\section{Efficient initialisation of iterative linear massive MIMO detectors using a stair matrix}

\author{
M. Albreem ${ }^{凶}$, M. Juntti and S. Shahabuddin
}

Several approximate matrix inversion methods have been used in linear massive MIMO uplink detectors where their convergence rate, performance, and complexity are greatly affected by the initial solution. In this Letter, the authors exploit a stair matrix, instead of a diagonal matrix, in initialising iterative linear minimum mean square error massive MIMO detector based on several approximate matrix inversion methods, namely, the Gauss-Seidel, successive over relaxation, Richardson iteration, and Newton iteration methods. Numerica results show a significant performance enhancement without burden of extra complexity using a stair matrix over a diagonal matrix in all methods.

Introduction: Massive MIMO is a key technology in the fifth generation wireless communications system both for uplink (UL) and downlink. It is an extension of the conventional small-scale multiuser MIMO transmission, where it employs a large number of antenna elements to serve simultaneously multiple users. Massive MIMO increases the diversity gain, provides link robustness, and improves the spectral and energy efficiencies. Along with the benefits, massive MIMO detectors for UL can suffer from a high-computational complexity: the maximum likelihood and the maximum a posteriori have exponential computational complexity [1]

In order to achieve a satisfactory balance between the performance and the complexity, a plethora of massive MIMO UL detection schemes have appeared in the literature. A comprehensive survey on detection techniques for massive MIMO is presented in [1]. Traditional linear detectors have been reinvigorated since the introduction of massive MIMO systems. A linear minimum mean square error (MMSE) detector is relatively simple to implement but it sustains a significant performance loss in highly loaded systems. It also computes the matrix inverse which exhibits a high-computational complexity. Two schemes have been proposed to minimise the linear detectors' complexity. The first scheme approximates iteratively the matrix inversion, rather than computing it. The Neumann series (NS) and Newton iteration (NI) methods are examples of the first scheme. The second scheme includes iterative methods to estimate the received signal by avoiding the matrix inversion, for instance, the Jacobi, Gauss-Seidel (GS), successive overrelaxation (SOR), and Richardson iteration (RI) methods.

The equalisation matrix in linear MMSE detector is diagonally dominant. Therefore, most of the existing linear MMSE detectors are utilising the diagonal matrix. In some cases, the diagonal matrix may not converge [2]. In [3], stair matrices and their generalisation are introduced with applications to iterative methods. In [2], the stair matrix is exploited in massive MIMO detectors based on the Jacobi and NS methods. It is shown that the convergence rate is accelerated by the use of a stair matrix compared to a diagonal matrix. Although the detector based on the Jacobi method can be easily implemented, it is neither robust nor as fast as the SOR and GS methods [2].

The good initialisation would impact the convergence rate and affect greatly both the complexity and performance. In this Letter, we propose to exploit the stair matrix in initialising the NI, SOR, GS, and RI methods for base station (BS) detectors. In the NI method, we used the stair matrix to estimate the initial matrix inversion of equalisation matrix $\left(\boldsymbol{A}_{(0)}^{-1}\right)$ and then it is used in iterations to approximate the inverse of matrix $\boldsymbol{A}$. In the SOR, GS, and RI methods, we utilised the stair matrix to compute the initial solution $\left(\hat{\boldsymbol{x}}_{(0)}\right)$.

Background: We consider a massive multi-user MIMO BS is serving $K$ single antenna UL users. The BS has a total of $N$ antennas where $N \gg K$. The $K$ users transmit their symbols individually and the symbol vector $\boldsymbol{x}=\left[x_{1}, x_{2}, \ldots \ldots, x_{K}\right]^{\mathrm{T}}$ is transmitted by all users. The BS receives a vector $\boldsymbol{y}=\left[y_{1}, y_{2}, \ldots . ., y_{N}\right]^{\mathrm{T}}$ corrupted by channel effects and noise. The relationship between $\boldsymbol{x}$ and $\boldsymbol{y}$ can be characterised as

$$
\boldsymbol{y}=\boldsymbol{H} \boldsymbol{x}+\boldsymbol{w},
$$

where $\boldsymbol{H}$ and $\boldsymbol{w}$ are the the channel matrix and $N \times 1$ additive white Gaussian noise, respectively. This model is generally used to derive the detection algorithm, where the channel state information and synchronisation are assumed to be perfect at the BS

Several methods have been proposed to avoid the computation of an exact matrix inversion of equalisation matrix $\boldsymbol{A}$

$$
\boldsymbol{A}=\boldsymbol{G}+\sigma^{2} \boldsymbol{I}_{K},
$$

where $\sigma^{2}, \boldsymbol{I}_{K}$, and $\boldsymbol{G}$ are the noise variance, $K \times K$ identity matrix, and Gram matrix or Gramian $\boldsymbol{G}=\boldsymbol{H}^{\mathrm{H}} \boldsymbol{H}$, respectively.

- The NI method approximates the matrix inversion of $\boldsymbol{A}$ as

$$
\boldsymbol{A}_{(n+1)}^{-1}=\boldsymbol{A}_{n}^{-1}\left(2 \boldsymbol{I}-\boldsymbol{A} \boldsymbol{A}_{n}^{-1}\right),
$$

where $n$ is the number of iterations. A common selection of the initial inversion is $\boldsymbol{A}_{(0)}^{-1}=\boldsymbol{D}^{-1}$, where $\boldsymbol{D}$ is the diagonal matrix.

- The SOR is an iterative method to estimate the signal as

$$
\hat{\boldsymbol{x}}_{(n+1)}=\left(\boldsymbol{D}-\frac{1}{\omega} \boldsymbol{L}\right)^{-1}\left(\boldsymbol{y}_{\mathrm{MF}}+\left(\left(1-\frac{1}{\omega}\right) \boldsymbol{D}+\frac{1}{\omega} \boldsymbol{U}\right) \hat{\boldsymbol{x}}_{(n)}\right),
$$

where $\omega$ is the relaxation parameter, $\boldsymbol{y}_{\mathrm{MF}}=\boldsymbol{H}^{\mathrm{H}} \boldsymbol{y}, \boldsymbol{U}$ and $\boldsymbol{L}$ are the upper triangular matrix and the lower triangular matrix, respectively.

- The GS method is a special case of the SOR method where $\omega=1$. The estimated signal is given as

$$
\hat{\boldsymbol{x}}_{(n+1)}=(\boldsymbol{D}-\boldsymbol{L})^{-1}\left(\boldsymbol{y}_{\mathrm{MF}}+\boldsymbol{U} \hat{\boldsymbol{x}}_{(n)}\right) .
$$

- The RI is an iterative method where $\boldsymbol{H}$ is utilised to find the estimated signal as

$$
\hat{\boldsymbol{x}}_{(n+1)}=\hat{\boldsymbol{x}}_{(n)}+\omega\left(\boldsymbol{y}_{\mathrm{MF}}-\boldsymbol{H} \hat{\boldsymbol{x}}_{(n)}\right) .
$$

Most of the existing linear MMSE detectors are utilising diagonal matrix $\boldsymbol{D}$. A stair matrix is a special tri-diagonal matrix where the offdiagonal elements on either the even or the odd row are zeros [3] Matrix $S$ is considered as a stair matrix if one of the following conditions is satisfied:

- $\boldsymbol{S}_{(i, i-1)}=0, \boldsymbol{S}_{(i, i+1)}=0$, where $i=2,4, \ldots, 2\left\lfloor\frac{K}{2}\right\rfloor$.

- $\boldsymbol{S}_{(i, i-1)}=0, \boldsymbol{S}_{(i, i+1)}=0$, where $i=1,3, \ldots, 2\left\lfloor\frac{K-1}{2}\right\rfloor+1$.

For instance, a $6 \times 6$ stair matrix has one of the following forms:

$S=\left[\begin{array}{cccccc}\times & \times & & & & \\ & \times & & & & \\ & \times & \times & \times & & \\ & & \times & & \\ & & \times & \times & \times \\ & & & & \times\end{array}\right]$ or $\boldsymbol{S}=\left[\begin{array}{ccccccc}\times & & & & & & \\ \times & \times & \times & & & \\ & & \times & & & \\ & \times & \times & \times & \\ & & & \times & \\ & & & & \times & \times\end{array}\right]$

Proposed methods:

- Linear detector based on the NI method and a stair matrix: the stair matrix $(\boldsymbol{S})$ can be extracted from matrix $\boldsymbol{A}$ as shown in background. The initial estimation of the matrix inverse is proposed to be

$$
\boldsymbol{A}_{(0)}^{-1}=\boldsymbol{S}^{-1}
$$

where $\boldsymbol{A}_{(0)}^{-1}$ will be used to initialise the iteration shown in (3), which converges quadratically to $\boldsymbol{A}^{-1}$ if

$$
\left\|\boldsymbol{I}-\boldsymbol{A} \boldsymbol{A}_{(0)}^{-1}\right\|<1 .
$$

The estimated signal $\hat{\boldsymbol{x}}$ is obtained as

$$
\hat{\boldsymbol{x}}=\boldsymbol{A}^{-1} \boldsymbol{y}_{\mathrm{MF}}
$$

Algorithm 1 shows the proposed iterative detector based on the NI method and a stair matrix. It includes the initialisation, iterations, and final estimation. An algorithm to find $\boldsymbol{S}^{-1}$ is given in [2]. 
Algorithm 1: Proposed detector based on the Newton method and a stair matrix

Input: $\boldsymbol{y}, \boldsymbol{H}, \sigma^{2}, n$

Output: estimated signal $\hat{\boldsymbol{x}}=\boldsymbol{M} \boldsymbol{y}_{\mathrm{MF}}$

Initialisation:

$\boldsymbol{A}=\boldsymbol{H}^{\mathrm{H}} \boldsymbol{H}+\sigma^{2} \boldsymbol{I}_{K}$

$\boldsymbol{y}_{\mathrm{MF}}=\boldsymbol{H}^{\mathrm{H}} \boldsymbol{y}$

$S=\operatorname{stair}(\boldsymbol{A})$

Initial estimation $\boldsymbol{M}_{(0)}=S^{-1}$

Iteration:

for $\mathrm{j}=0: 1: n$

$\boldsymbol{M}_{(j+1)}=\boldsymbol{M}_{(j)}\left(2 \boldsymbol{I}-\boldsymbol{A} \boldsymbol{M}_{(j)}\right)$;

end

Return $M$.

- Linear detector based on iterative methods: in [2], the diagonal matrix $(\boldsymbol{D})$ is replaced by the stair matrix in a detector based on the Jacobi method where it improves the performance. Detectors based on other iterative methods such as the SOR and GS methods depend on $\boldsymbol{D}, \boldsymbol{U}$, and $\boldsymbol{L}$. A common selection of the initial solution is $\hat{\boldsymbol{x}}_{(0)}=\boldsymbol{D}^{-1} \boldsymbol{y}_{\mathrm{MF}}$. Herein, we propose to use the stair matrix in finding the initial solution $\left(\hat{\boldsymbol{x}}_{(0)}\right)$ for the SOR, GS, and RI methods as

$$
\hat{\boldsymbol{x}}_{(0)}=\boldsymbol{S}^{-1} \boldsymbol{y}_{\mathrm{MF}} .
$$

The core of iterative methods will not be changed. Algorithm 2 shows the iterative methods using the proposed initialisation.

Algorithm 2: Proposed detector based on iterative methods (SOR, GS, and RI) and a stair matrix

Input: $\boldsymbol{y}, \boldsymbol{H}, \sigma^{2}, n, \omega$

Output: Estimated signal $\hat{\boldsymbol{x}}$

Initialisation:

$\boldsymbol{A}=\boldsymbol{H}^{\mathrm{H}} \boldsymbol{H}+\sigma^{2} \boldsymbol{I}_{K}$

$\boldsymbol{D}=\operatorname{diag}(\boldsymbol{A}), \boldsymbol{U}=-\operatorname{triu}(\boldsymbol{A}), \boldsymbol{L}=-\operatorname{tril}(\boldsymbol{A})$

$\boldsymbol{y}_{\mathrm{MF}}=\boldsymbol{H}^{\mathrm{H}} \boldsymbol{y}$

$S=\operatorname{stair}(\boldsymbol{A})$

Initial estimation $\hat{\boldsymbol{x}}_{(0)}=\boldsymbol{S}^{-1} \boldsymbol{y}_{\mathrm{MF}}$

Iteration:

for $j=0: 1: n$

Apply SOR, GS, or RI using (4), (5) or (6), respectively

end

Return $\hat{x}$.

Complexity analysis: The computation of $S^{-1}$ requires $K$ real number of divisions and $3(K-1)$ real number of multiplications while the computation of $\boldsymbol{D}^{-1}$ requires $K$ real number of divisions. Although the use of a stair matrix increases the number of multiplications by $3(K-1)$, this complexity increment is trivial. For instance, in a $16 \times 128 \mathrm{MIMO}$ and $n=3$, the number of multiplications required in the GS method using a stair matrix is 3117 multiplications while it requires 3072 multiplications when using the diagonal matrix. Table 1 presents the computational complexity in terms of required multiplications, which is the dominant operation in the total computational complexity. However, the complexity to compute $S^{-1}$ is $\mathcal{O}(K)$, which is the same order of the complexity to compute $\boldsymbol{D}^{-1}$.

Table 1: Complexity comparison among initialised detectors

\begin{tabular}{|c|c|}
\hline Method & Number of multiplications \\
\hline NI & $2(n-1) K^{3}+N K^{2}+K(N+3)-3$ \\
\hline RI & $4 n K^{2}+K(2 n+3)-3$ \\
\hline SOR & $4 n K^{2}+K(n+3)-3$ \\
\hline GS & $4 n K^{2}+3(K-1)$ \\
\hline Jacobi & $2 n K(2 K-1)$ \\
\hline NS & $(n-2) K^{3}+N K^{2}+K(N+3)-3$ \\
\hline
\end{tabular}

Numerical results: In this section, the performance of a detector based on the proposed initialisation of the NI, GS, SOR, and RI methods will be presented in BER versus the signal-to-noise ratio (SNR). We consider independent and identically distributed (i.i.d) Gaussian channels, the
MIMO size is $16 \times 128$, the modulation scheme is $64-\mathrm{QAM}$, and several iterations are conducted.

In order to investigate the condition in (8), we generate $10^{4}$ random $\boldsymbol{H}$ and the stair matrix $\boldsymbol{S}$ is extracted from each $\boldsymbol{H}$. As shown in Table 2, the convergence condition in (8) is satisfied for all extracted $\boldsymbol{S}$ and the stair matrix is applicable to be used in such iterative methods.

Table 2: Statistics of $\left\|\boldsymbol{I}-\boldsymbol{A} \boldsymbol{A}_{(0)}^{-1}\right\|$ in (8) for $10^{4}$ channel realisations

\begin{tabular}{|c|c|c|c|}
\hline Mean & Median & Standard deviation & Prob. quadratic convergence \\
\hline 0.6305 & 0.6276 & 0.2510 & 1 \\
\hline
\end{tabular}

Fig. 1 illustrates the performance of a detector based on approximate matrix inversion methods using both the diagonal matrix and the stair matrix. In all iterations, a detector initialised by a stair matrix outperforms others. For instance, at SNR $=17 \mathrm{~dB}$ and $n=1$, the BER is $10^{-4}$ and $10^{-2}$ for the GS-based detector using a stair matrix and diagonal matrix, respectively. It also shows that the worst performance has been obtained by the RI-based detector.
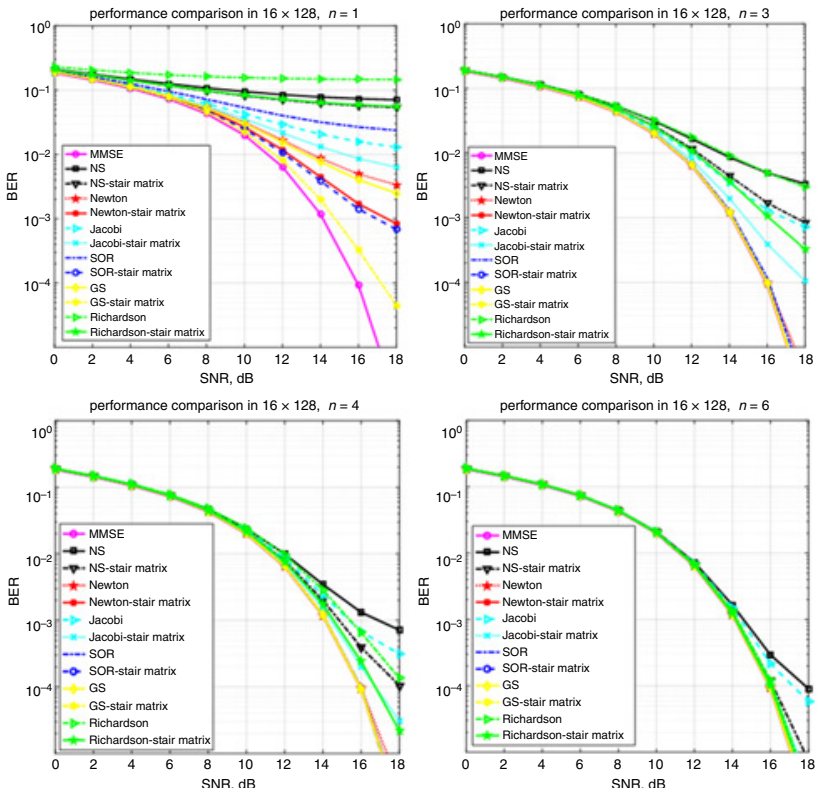

Fig. 1 Performance of detector based on several approximate matrix inversion methods and stair matrix for $16 \times 128$ massive MIMO system and $64-Q A M$

Fig. 2 compares the performance-complexity profile of initialised iterative linear detectors to achieve BER $=10^{-3}$. The detector based on the GS method achieves the target BER at SNR $=15 \mathrm{~dB}$ with the lowest complexity. The detector based on the NS and NI methods requires a high complexity to achieve the target BER.

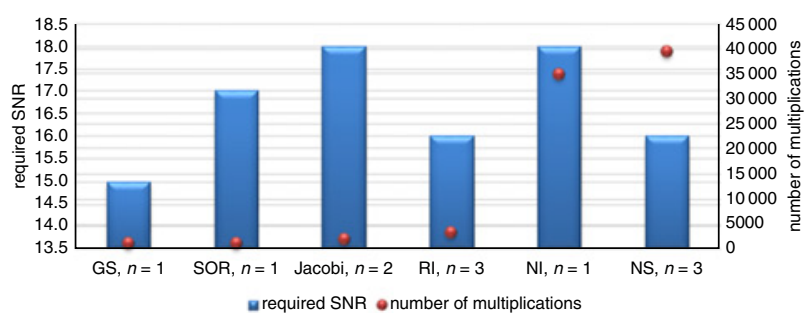

Fig. 2 Performance-complexity trade-off to achieve BERBER $=10^{-3}$

Conclusion: A stair matrix is used to initialise the linear massive MIMO detector based on the NI, GS, SOR, and RI methods. Proposed initialisation using a stair matrix achieved a performance enhancement without a burden of extra complexity. A detector based on the SOR and GS methods achieved the target performance with the lowest complexity.

Acknowledgments: This research has been financially supported by Academy of Finland 6Genesis Flagship (grant no. 318927), Nokia 
Foundation Centennial Grant, A'Sharqiyah University Research Visits Support Fund, and The Research Council of Oman (grant no. BFP/ RGP/ICT/18/079).

(C) The Institution of Engineering and Technology 2020

Submitted: 30 August 2019

One or more of the Figures in this Letter are available in colour online.

M. Albreem (Department of Electronics and Communications Engineering, A'Sharqiyah University, Ibra 400, Oman)

凶 E-mail: mahmoud.albreem@asu.edu.om

M. Juntti (Centre for Wireless Communications, University of Oulu, Oulu 90014, Finland)

S. Shahabuddin (Mobile Networks, Nokia, Oulu 90620, Finland)

S. Shahabuddin: Also with Centre for Wireless Communications, University of Oulu, Oulu 90014, Finland

\section{References}

1 Albreem, M.A., Juntti, M., and Shahabuddin, S.: 'Massive MIMO detection techniques: a survey', Commun. Surv. Tutor., 2019, pp. 1-25, doi: 10.1109/COMST.2019.2935810

2 Jiang, F., Li, C., Gong, Z., et al.: 'Stair matrix and its applications to massive MIMO uplink data detection', Trans. Commun., 2018, 66, (6), pp. 2437-2455

$3 \mathrm{Lu}, \mathrm{H} .:$ 'Stair matrices and their generalizations with application to iterative methods I: a generalization of the successive overrelaxation method', SIAM J. Numer. Anal., 1999, 37, (1), pp. 1-17 